SERIE B - INFORMATIK

\title{
Generalized Davenport-Schinzel Sequences
}

\author{
Martin Klazar* \\ Pavel Valtr** \\ B $94-04$ \\ January 1994
}

\begin{abstract}
The extremal function $E x(u, n)$ (introduced in the theory of Davenport-Schinzel sequences in other notation) denotes for a fixed finite alternating sequence $u=a b a b a \ldots$ the maximum length of a finite sequence $v$ over $n$ symbols with no immediate repetition which does not contain $u$. Here (following the idea of J. Nešetruil) we generalize this concept for arbitrary sequence $u$. We summarize the already known properties of $\operatorname{Ex}(u, n)$ and we present also two new theorems which give good upper bounds on $E x(u, n)$ for $u$ consisting of (two) smaller subsequences $u_{i}$ provided we have good upper bounds on $E x\left(u_{i}, n\right)$. We use these theorems to describe a wide class of sequences $u$ ("linear sequences") for which $E x(u, n)=O(n)$. Both theorems are used for obtaining new superlinear upper bounds as well. We partially characterize linear sequences over three symbols. We also present several problems about $E x(u, n)$.
\end{abstract}

Key words: Davenport-Schinzel sequence, extremal problem, maximum length

*Department of Applied Mathematics, Charles University, Malostranské nám. 25, 11800 Praha 1, Czech Republic, E-mail: klazar@cspguk11.bitnet.

**Department of Applied Mathematics, Charles University, Malostranské nám. 25, 11800 Praha 1, Czech Republic, and Graduiertenkolleg "Algorithmische Discrete Mathematik", Fachbereich Mathematik, Freie Universität Berlin, Takustrasse 9, 14195 Berlin, Germany, E-mail: valtr@inf.fu-berlin.de. Supported by

"Deutsche Forschungsgemeinschaft", grant We 1265/2-1. 


\section{Introduction}

In this paper we shall investigate the maximum length $E x(u, n)$ of finite sequences over $n$ symbols not containing a fixed sequence $u$. We search for sequences $u$ for which there is a linear upper bound on $E x(u, n)$. We call them linear sequences.

First we give a brief informal overview of results concerning extremal problems of this type (belonging to a branch which could be called "Extremal theory of sequences"). After that all necessary definitions will be introduced. The first section concludes by the formulation of our main result: we present two operations which enable us to derive upper bounds on $E x(u, n)$ from upper bounds for shorter $u$. In the second section we summarize the properties of $\operatorname{Ex}(u, n)$ which are useful in the proofs. In the third section we show four applications of our operations: we prove the linearity of certain relatively complicated sequences, we show on examples how to derive nonlinear upper bounds on $E x(u, n)$, we discuss the linearity of sequences over three symbols and we describe which linear sequences we are able to obtain at present. The remaining two sections are devoted to the proofs of the main theorems. In the second and in the third sections we list some problems which might stimulate further research in this area.

\subsection{History}

Davenport-Schinzel sequences are finite sequences over $n$ symbols with no immediate repetition of the same symbol which contain no five-term alternating subsequence (or, more generally, no alternating subsequence of the length $s$ ). Davenport and Schinzel posed [4] the problem to estimate the maximum length of such sequences. They proved in [4] Ex $(a b a b a, n)=O(n \cdot \log n)(E x(a b a b a, n)=O(n \cdot \log n / \log \log n)$ in [5]) and $E x(a b a b a b \ldots, n)=O(n . \exp (\sqrt{n})$ ( $s$ fixed) which was improved by Szemerédi [14] to $\operatorname{Ex}(a b a b a b \ldots, n)=O\left(n \cdot \log ^{*} n\right)$. As usual $\log ^{*} n$ denotes the minimum number of iterations of the power function $2^{m}$ (starting with $m=1$ ) which are needed to get a number bigger or equal to $n$. However, the problem whether $E x(a b a b a, n)=O(n)$ remained open until 1986 when it was answered [7] by Hart and Sharir in the negative: they proved $E x(a b a b a, n)=\Theta(n . \alpha(n))$ where $\alpha(n)$ is the inverse to the Ackerman function and goes to infinity but extremely slowly. Later simpler constructions proving $\operatorname{Ex}(a b a b a, n)=\Omega(n . \alpha(n))$ were found ( $[11],[15]$ ). M. Sharir [12] derived the upper bounds (ababab... is of the length $s$ )

$$
E x(a b a b a b \ldots, n)=O\left(n . \alpha(n)^{O\left(\alpha(n)^{s-5}\right)}\right) .
$$

Agarwal, Sharir and Shor [3] found almost tigh upper and lower bounds:

$$
\begin{array}{ll}
\operatorname{Ex}(a b a b a b \ldots, n) \leq n .2^{(\alpha(n))^{\frac{s-5}{2}} \log _{2} \alpha(n)+C_{s}(n)} & \text { for } s \geq 5 \text { odd } \\
\operatorname{Ex}(a b a b a b \ldots, n) \leq n .2^{(\alpha(n))^{\frac{s-4}{2}}+C_{s}(n)} & \text { for } s \geq 6 \text { even } \\
\operatorname{Ex}(a b a b a b \ldots, n)=\Omega\left(n .2^{K_{s} \cdot(\alpha(n))^{\frac{s-4}{2}}+Q_{s}(n)}\right) & \text { for } s \geq 6 \text { even }
\end{array}
$$


where $K_{s}=\frac{1}{\left(\frac{s-4}{2}\right) !}$ and the functions $C_{s}(n)$ and $Q_{s}(n)$ are asymptoticaly smaller then the main terms in the exponent. For $s=6$ they found a stronger estimate $\operatorname{Ex}(a b a b a b, n)=\Theta\left(n .2^{\alpha(n)}\right)$.

Füredi and Hajnal [6] investigated a similar problem what is the maximum number of 1 's in a 0-1 matrix of the size $n \times n$ if some configurations are forbidden.

The primary motivation of Davenport-Schinzel sequences was geometrical and now they play an important role in computational geometry. See the books [2] and [13] for more information and references.

The function $E x(u, n)$ extending functions $E x(a b a b a, n) \operatorname{resp} . E x(a b a b a b \ldots, n)$ was defined in [1]. Note here that this definition which follows in the next subsection was suggested by J. Nešetruil. Some other results from [1] will be mentioned in the second section. In [8] it was proved that

$$
\operatorname{Ex}(u, n)=n .2^{O\left(\alpha(n)^{|u|-4}\right)}
$$

( $|u|$ denotes the length of $u$ ) for any fixed finite sequence $u$. Thus, from the practical point of view, $E x(u, n)$ has a "linear" upper bound for any $u$. In this paper we study the question for which $u$ actually $E x(u, n)=O(n)$ and we give a partial answer to it. It is easy to prove that it holds for $u=a b a b$ but it is not so easy to prove the same thing for $u=a a b b a a b b$. The more general sequence

$$
x_{1} \ldots x_{1} x_{2} \ldots x_{2} \ldots x_{k} \ldots x_{k} x_{1} \ldots x_{1} x_{2} \ldots x_{2} \ldots x_{k} \ldots x_{k}
$$

is linear as well ( [9]) but here we prove stronger results.

\subsection{Definitions}

For any finite sequence $u$ we denote by $|u|$ the length of $u$, by $S(u)$ the set of all symbols occuring in $u$ and by $\|u\|$ the size of $S(u)$. Thus $\|u\| \leq|u|$ for all $u$. The sequences for which equality is achieved are called chains. Hence in chains no symbol repeats.

Two sequences $u=a_{1} a_{2} \ldots a_{n}$ and $v=b_{1} b_{2} \ldots b_{n}$ of the same length are equivalent if there exists a bijection $f: S(u) \rightarrow S(v)$ such that $f\left(a_{i}\right)=b_{i}$ for all $i=1,2, \ldots, n$. Thus $u$ and $v$ coincide after a renaming of symbols. The notation $u \subseteq v$ means that $u$ is a subsequence of $v$. We say that $v$ contains $u$, formally $u \prec v$, if $u$ is equivalent to some $t, t \subseteq v$.

We shall refer to the occurrences of a symbol $a \in S(u)$ in the sequence $u$ as to a-occurrences. The sequence $u=a a \ldots a,|u|=i$ is denoted shortly by $a^{i}$.

The mirror image $a_{n} \ldots a_{2} a_{1}$ of the sequence $u=a_{1} a_{2} \ldots a_{n}$ is denoted by $\bar{u}$. The sequence $u=a_{1} a_{2} \ldots a_{m}$ is called $k$-regular if $a_{i}=a_{j}, i \neq j$ implies $|i-j| \geq k$. Thus any at most $k$ consecutive elements in $u$ are different to each other. DavenportSchinzel sequences are 2-regular.

\section{Definition 1.2.1}

$$
E x(u, n)=\max \{|v| \mid u \nprec v, v \text { is }\|u\| \text {-regular, }\|v\| \leq n\}
$$


where $u$ is a fixed sequence, $v$ is arbitrary sequence and $n \geq 1$ is an integer.

We investigate the behaviour of this function for $u$ fixed and $n$ growing to infinity. Sometimes a more general definition will be useful:

\section{Definition 1.2.2}

$$
\operatorname{Ex}(u, n, l)=\max \{|v| \mid u \nprec v, v \text { is l-regular, }\|v\| \leq n\}
$$

where $l \geq\|u\|$ is an integer.

Obviously $E x(u, n)=\operatorname{Ex}(u, n, l)=\|u\|-1$ for any chain $u$ and $E x(u, n, l) \geq n$ for any nonchain $u$. Also $E x\left(a^{i}, n\right)=(i-1) n$ and $E x(a b a b, n)=2 n-1$. Our interest is focused on the set

\section{Definition 1.2.3}

$$
\operatorname{Lin}=\{u \mid \operatorname{Ex}(u, n)=O(n)\} .
$$

We call its elements linear sequences. Other sequences, for instance $a b a b a$, are called nonlinear.

\subsection{Results}

The following two theorems are the main result of this paper.

Theorem A Suppose that $u=u_{1} a^{2} u_{2}$ and $v$ are two sequences such that $S(u) \cap$ $S(v)=\emptyset$ and $a$ is a symbol. If $v$ is not a chain then $\operatorname{Ex}\left(u_{1} a v a u_{2}, n\right)=O(E x(v, 2 E x(u, n)))$. In the case $v$ is a chain $\operatorname{Ex}\left(u_{1} a v a u_{2}, n\right) \leq E x(u, n)$ holds.

Theorem B Suppose $a, b$ are two symbols and $u=u_{1} a^{2} u_{2} a$ is a sequence such that $b \notin S(u)$. Then $\operatorname{Ex}\left(u_{1} a b^{i} a u_{2} a b^{i}, n\right)=\Theta(\operatorname{Ex}(u, n))$ for any $i \geq 1$.

We are interested in linear sequences. For them it follows immediately

Consequence $\mathbf{A}$ Let $u, v \in \operatorname{Lin}$ be as in Theorem A. Then $u_{1} a v a u_{2} \in \operatorname{Lin}$.

Consequence B Let $u \in \operatorname{Lin}$ and $b$ be as in Theorem B. Then $u_{1} a b^{i} a u_{2} a b^{i} \in \operatorname{Lin}$ for all $i \geq 1$.

We use both consequences in the obvious manner: by repeated applications of both transformations $u, v \rightarrow u_{1} a v a u_{2}$ and $u \rightarrow u_{1} a b^{i} a u_{2} a b^{i}$ we can generate a wide class of linear sequences. 


\section{Properties of $\operatorname{Ex}(\mathrm{u}, \mathrm{n})$}

Fact 2.1 Suppose two sequences $u$ and $v$ and two integers $l>k \geq\|u\|$ are given. Then for any $n \geq 1$

1. $\operatorname{Ex}(u, n, k)$ is finite and $\operatorname{Ex}(u, n, k)=O\left(|u| \cdot\|u\| . n^{\|u\|}\right)$.

D. $\operatorname{Ex}(u, n, l) \leq E x(u, n, k) \leq(E x(u, l-1, k)+1) \operatorname{Ex}(u, n, l)$.

3. If $v \prec u$ then $E x(v, n)=O(E x(u, n))$. Especially if $u$ is linear then $v$ is linear too.

4. $\operatorname{Ex}(u, n)=\operatorname{Ex}(\bar{u}, n)$.

Proof: The first claim follows from Pigeon-Hole argumentation (we mentioned in the Introduction that a far stronger estimate holds ( [8]) ). The second inequality in the second claim is obtained by deleting occurrences by a a greedy algorithm. For both proofs we refer to [1]. The first inequality as well as the fourth claim are obvious. We prove the third claim. Suppose $w$ is a $\|u\|$-regular sequence not containing $v$. Hence it cannot contain $u$ and therefore $\operatorname{Ex}(v, n,\|u\|) \leq E x(u, n,\|u\|)=\operatorname{Ex}(u, n)$. The second inequality in 2. yields the estimate

$$
E x(v, n)=E x(v, n,\|v\|)=O(E x(v, n,\|u\|)=O(E x(u, n)) .
$$

The reason why we exclude from the following considerations chains is that the extremal function of any chain is constant and hence for these singular sequences the nice estimates are not valid.

Fact 2.2 Suppose $u, v$ and $w$ are three sequences, $i \geq 2, j \geq 2$ are two integers and $a$ is a symbol such that au is not a chain. Then for $n \geq 1$

1. $\operatorname{Ex}\left(a^{i} u, n\right)=\Theta(\operatorname{Ex}(a u, n)), E x\left(u a^{i}, n\right)=\Theta(E x(u a, n))$.

2. $\operatorname{Ex}\left(w a^{j} v, n\right)=\Theta\left(\operatorname{Ex}\left(w a^{2} v, n\right)\right)$.

Proof: See in [1].

Let us reformulate Fact 2.2. Suppose the sequence $u$ is written in the "exponential form" $u=a_{1}^{i_{1}} a_{2}^{i_{2}} \ldots a_{r}^{i_{r}}, a_{i} \neq a_{i+1}, i_{j} \geq 1$ (note that $i_{1}=i_{2}=\ldots=i_{r}=1$ iff $u$ is 2-regular). The reduced sequence is defined by $\operatorname{red}(u)=a_{1}^{j_{1}} a_{2}^{j_{2}} \ldots a_{r}^{j_{r}}$ where $j_{1}=j_{r}=1$ and $j_{k}=\min \left\{2, i_{k}\right\}$ for $1<k<r$. The fully reduced sequence is defined by $\operatorname{fred}(u)=a_{1} a_{2} \ldots a_{r}$.

Fact 2.3 $E x(u, n)=\Theta(E x(\operatorname{red}(u), n))$ for any sequence $u$ and any integer $n \geq 1$ provided that red $(u)$ is not a chain. 
Proof: Follows from the previous Fact 2.2.

There is the question whether $\operatorname{Ex}(u, n)=\Theta(\operatorname{Ex}(\operatorname{fred}(u), n))$ (suppose $\operatorname{fred}(u)$ is not a chain) which is equivalent to the following problem.

Problem 2.1 Does $\operatorname{Ex}\left(u a^{2} v, n\right)=O(\operatorname{Ex}(u a v, n))$ hold for any symbol a and all sequences $u, v$ (provided uav is not a chain)?

Fact 2.4 Suppose $i \geq 1$ is an integer, $u$ is a sequence over two symbols $(\|u\| \leq 2)$ and $a, b$ are two symbols. Then

1. $a^{i} b^{i} a^{i} b^{i}$ is linear.

2. ababa is nonlinear.

3. $u$ is nonlinear iff $a b a b a \prec u$.

Proof: For the proof of the first statement we refer to [1]. Or apply Consequence B to the sequence $a^{2 i}$. The second claim is proved in [7].

We deduce the last statement from the first two. If $a b a b a \prec u$ then clearly $u$ is nonlinear. If the sequence $u$ is over two symbols and does not contain ababa then clearly $u=a^{m_{1}} b^{m_{2}} a^{m_{3}} b^{m_{4}}$ where $m_{i} \geq 0$. Thus $u \prec a^{m} b^{m} a^{m} b^{m}$ for $m=\max \left(m_{i} \mid i=\right.$ $1,2,3,4)$ and $u$ is linear according to 1 . and according to 3 . of Fact 2.1 .

\section{Applications of Theorems A and B}

\subsection{Linearity of $a b c d c b a b c d$}

The sequence $w(k, i)=a_{1}^{i} a_{2}^{i} \ldots a_{k}^{i} a_{1}^{i} a_{2}^{i} \ldots a_{k}^{i}$ which we mentioned in Introduction is a $k$-symbol analog to $a^{i} b^{i} a^{i} b^{i}$. It was proved in [9] by methods different from those presented here that $w(k, i)$ is linear. Now we prove a stronger result.

Theorem 3.1.1 The sequence $z(k, i)=a_{1}^{i} a_{2}^{i} \ldots a_{k-1}^{i} a_{k}^{i} a_{k-1}^{i} \ldots a_{2}^{i} a_{1}^{i} a_{2}^{i} \ldots a_{k-1}^{i} a_{k}^{i}$, where the symbols $a_{1}, a_{2}, \ldots a_{k}$ are mutually distinct, is linear for all $i, k \geq 1$.

Proof: By induction on $k$. For $k=1$ the sequence $z(1, i)=a^{2 i}$ is linear. Suppose that the assertion holds for $k>1$. Thus $a_{1}^{i} a_{2}^{i} \ldots a_{k-1}^{i} a_{k}^{2 i} a_{k-1}^{i} \ldots a_{2}^{i} a_{1}^{i} a_{2}^{i} \ldots a_{k-1}^{i} a_{k}^{i} \prec$ $z(k, 2 i)$ is linear because $z(k, 2 i)$ is a linear sequence. Applying Consequence B we conclude that $z(k+1, i)$ is linear as well. 


\subsection{Superlinear bounds}

Despite the fact that we stress the linear case here we cannot resist the temptation to present an application of our theorems to nonlinear sequences. The difficulty is that the strong superlinear bounds of [7] and [3] were derived only for the 2regular case and we need a bit more here. However, it may be checked that the

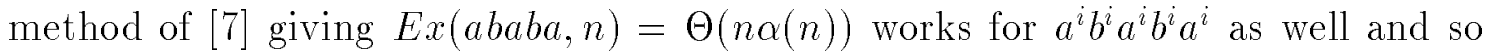
$E x\left(a^{i} b^{i} a^{i} b^{i} a^{i}, n\right)=\Theta(n \alpha(n))$. We present two examples of new strong superlinear upper bounds. It is possible to derive other similar results.

\section{Theorem 3.2.1}

$$
\Omega(n \alpha(n))=\operatorname{Ex}(a b a c d c d c a b a, n)=O\left(n \alpha^{2}(n)\right)
$$

Proof: The lower bound follows trivially from $a b a b a \prec a b a c d c d c a b a$. The upper bound follows from Theorem A:

$$
\begin{gathered}
E x(a b a c d c d c a b a, n)=O\left(E x\left(a b a b a, 2 E x\left(a b a^{2} b a, n\right)\right)\right)= \\
=O(O(n \alpha(n)) \cdot \alpha(O(n \alpha(n))))=O\left(n \alpha^{2}(n)\right)
\end{gathered}
$$

where $\alpha(O(n \alpha(n)))=O(\alpha(n))$ follows from the extreme slow growth of $\alpha(n)$.

\section{Theorem 3.2.2}

$$
E x(a b a c d c a b a c d, n)=\Theta(n \alpha(n))
$$

Proof: This result follows from Theorem B:

$$
E x(a b a c d c a b a c d, n)=\Theta\left(E x\left(a b a c^{2} a b a c, n\right)\right)=\Theta\left(E x\left(a b a^{2} b a, n\right)\right)=\Theta(n \alpha(n)) .
$$

\subsection{Linear sequences over three symbols}

The third point of Fact 2.4 gives a complete characterization of linear sequences over two symbols. We would like to obtain a similar characterization for the whole set Lin of linear sequences but this seems to be a hard task. Though we are unable to decide linearity even in the slightly more general case of sequences over three symbols, we give for these sequences a characterization theorem which puts away only few sequences as undecided.

Theorem 3.3.1 Suppose $u$ is a sequence over three symbols $(\|u\| \leq 3)$ and neither $u$ nor $\bar{u}$ contains any of the three sequences

$$
\text { ababa } \quad c a b a b c b \quad a c b a b c b \text {. }
$$

Then $u$ is a linear sequence. 
Proof: Suppose that $a b a b a \nprec u$. It is not difficult to show that any 2-regular sequence $u, a b a b a \nprec u,\|u\| \leq 3$ is contained in one of three sequences $u_{1}=a b a b c b c, u_{2}=$ $a b c b a b c$ and $u_{3}=a c a b a b c b$. Thus $a b a b a \nprec u,\|u\| \leq 3$ implies $u \prec u_{1}(i)$ or $u \prec u_{2}(i)$ or $u \prec u_{3}(i)$ (for large $i$ ) where $u_{1}(i)=a^{i} b^{i} a^{i} b^{i} c^{i} b^{i} c^{i}$, similarily for $u_{2}(i)$ and $u_{3}(i)$. But, as may be deduced from Consequence B and Fact 2.4.1., $u_{1}(i)$ and $u_{2}(i)$ are linear (actually $\left.u_{2}(i)=z(3, i)\right)$. Hence $u$ is linear or $u \prec u_{3}(i)$.

Using Consequences $\mathrm{A}$ and $\mathrm{B}$ it may be proved that any subsequence of $u_{3}(i)$ which does not contain any of the four sequences $s_{1}=c a b a b c b, s_{2}=a c b a b c b, s_{3}=a c a b a c b$ and $s_{4}=a c a b a b c$ is linear. Thus $u$ is linear or $u \succ s_{j}$ for some $j \in\{1,2,3,4\}$. But $s_{1}$ is equivalent to $\overline{s_{4}}$ and $s_{2}$ is equivalent to $\overline{s_{3}}$. We are finished.

In the opposite direction we are able to say only that $a b a b a \prec u$ implies the nonlinearity of $u$. The linearity of three sequences over three symbols $u_{3}=a c a b a b c b, s_{1}=$ $c a b a b c b$ and $s_{2}=a c b a b c b$ remains open.

Problem 3.3.1 Are $u_{3}, s_{1}$ and $s_{2}$ resp. $u_{3}(i), s_{1}(i)$ and $s_{2}(i)$ linear?

\subsection{How to get many linear sequences}

We conclude this section by a compact description of the widest class of sequences $M$ such that $M \subseteq$ Lin may be proved from above.

Let $M$ be the minimal (to inclusion) set of sequences satisfying the following rules

1. $a^{i} \in M$ for any symbol $a$ and any integer $i, i \geq 1$.

2. If $u=u_{1} a^{2} u_{2}$ and $v$ ( $a$ is a symbol) are two sequences of $M$ such that $S(u) \cap$ $S(v)=\emptyset$ then $u_{1}$ avau $u_{2}$ lies in $M$ as well.

3. If $u=u_{1} a^{2} u_{2} a$ ( $a$ is a symbol) is a sequence of $M$ and $b, b \notin S(u)$ is a new symbol then $u_{1} a b^{i} a u_{2} a b^{i}$ lies in $M$ for any $i \geq 1$ as well.

4. If $u$ is a sequence of $M$ and $v, v \prec u$ is another sequence then $v$ lies in $M$ as well.

5. If $u$ is a sequence of $M$ then $\bar{u}$ lies in $M$ as well.

Then

Theorem 3.4.1 $M \subseteq \operatorname{Lin}$

Proof: We see immediately that the first and the last two rules preserve linearity and hence it suffices to prove only that the set Lin is closed on the second rule as on the third one. But this is exactly the statement of Consequence A and B.

We present, as a concrete example of an application of the previous theorem, three linear sequences. The reader will be surely able to establish how the previous five 
rules were used to derive these sequences as well as (s) he will be able to obtain many others.

\section{Example}

The following three sequences belong to $M$ and therefore they all are linear. ababcbcdcdedefefgfg ccaaccaabbdefedefbabb ccaaccabgggbdefedefbbbaabbgg

It is worth noting that $u \in M$ iff $f r e d(u) \in M$. Hence the answer to Problem 2.1 is affirmative if we restrict ourselves to $M$. This supports the conjecture that the change of any exponent in $u$ does not influence (except the trivial case of a chain) the growth rate of $E x(u, n)$.

Problem 3.4.1 Characterize the set Lin. Are there D-regular linear sequences over $n$ symbols which are longer than $3 n-2$ ?

This bound is achieved for instance by $z(n, 1)$ or by the sequences which are constructed as the first sequence in the example above.

Problem 3.4.2 Suppose $B$ is the set of all minimal (to $\prec)$ nonlinear sequences. What can be said about the elements of $B$ ? Is $B$ finite?

The set $\operatorname{Lin}$ is closed to $\prec(u \prec v \in \operatorname{Lin} \Rightarrow u \in \operatorname{Lin})$ and thus $B$ characterizes Lin: $u \notin \operatorname{Lin}$ iff there is a sequence $v$ that $v \in B, v \prec u$. The set $B$ serves for Lin as a collection of "forbidden pictures". An immediate observation is that ababa $\in B$. Another observation, nontrivial, is that the set $B$ must contain at least two elements: the simple and nice construction of [15] proving $E x(a b a b a, n)=\Omega(n \alpha(n))$ proves also implicitely $E x(w, n)=\Omega(n \alpha(n))$ where $w=$ abcbadadbcd but ababa $\nprec w$. Thus there is a sequence $u^{*}, u^{*} \neq a b a b a, u^{*} \prec w, u^{*} \in B$. For details we refer to [10].

\section{Proof of Theorem A}

Suppose $a$ is a symbol and $u=u_{1} a^{2} u_{2}$ and $v$ are two sequences such that $S(u) \cap$ $S(v)=\emptyset$. We denote by $t$ the sequence $u_{1} a v a u_{2}$.

We start with the simpler case of Theorem A when $v$ is a chain. Suppose $w$ is a $\|t\|$-regular sequence not containing $t$. We show that $w$ cannot even contain $u$. Let $s \subseteq w$ be equivalent to $u$. The $\|t\|$-regularity of $w$ implies that there occur $\|v\|$ distinct symbols between the two " $a$ ' $s$ " in $s$ which are not elements of $S(s)$. Thus $t \prec w$ which is a contradiction. We get an inequality $\operatorname{Ex}(t, n,\|t\|) \leq E x(u, n,\|t\|)$. Hence $\operatorname{Ex}(t, n)=\operatorname{Ex}(t, n,\|t\|) \leq E x(u, n,\|t\|) \leq \operatorname{Ex}(u, n,\|u\|)=\operatorname{Ex}(u, n)$.

Before proving the first part of Theorem A we give an auxiliary lemma. We say that a nondecreasing integral function $f:\{1,2, \ldots\} \rightarrow\{1,2, \ldots\}$ is big if $\sum_{i} f\left(n_{i}\right) \leq$ $c f\left(\sum n_{i}\right)$ for some fixed constant $c$ for any sum of integers $\sum_{i} n_{i}$. 
Lemma 4.1 The function $\operatorname{Ex}(u, n)$ is big for any nonchain $u$.

Proof: We call in this proof a sequence $u$ irreducible if there does not exist a nontrivial decomposition $u=u_{1} u_{2}$ such that $S\left(u_{1}\right) \cap S\left(u_{2}\right)=\emptyset$. Otherwise we call it reducible. It is easy to see that $\operatorname{Ex}(u, n)$ is big with constant $c=1$ for any irreducible $u$. Indeed, if $n=n_{1}+n_{2}+\cdots+n_{m}$ then by concatenation of $m\|u\|$ regular sequences $v_{i}, u \nprec v_{i},\left\|v_{i}\right\| \leq n_{i},\left|v_{i}\right|=E x\left(u, n_{i}\right), S\left(v_{i}\right) \cap S\left(v_{j}\right)=\emptyset$ we get a $\|u\|$-regular sequence $v=v_{1} v_{2} \ldots v_{m},\|v\| \leq n$ not containig $u$ which proves the desired inequality. To manage the case of reducible sequences we need the following claim.

Claim $E x\left(u_{1} u_{2}, n\right)=O\left(E x\left(u_{1}, n\right)+E x\left(u_{2}, n\right)\right)$ for any two sequences $u_{1}, u_{2}$, $S\left(u_{1}\right) \cap S\left(u_{2}\right)=\emptyset$ except when $\left|u_{1}\right|=\left|u_{2}\right|=1$.

Proof: Suppose $v$ is $\left\|u_{1}\right\|+\left\|u_{2}\right\|$-regular, $|v| \geq c\left(E x\left(u_{1},\|v\|\right)+E x\left(u_{2},\|v\|\right)\right)$ for some large fixed constant $c$. We show that this implies $u_{1} u_{2} \prec v$. We split $v=v_{1} v_{2},\left|v_{1}\right| \doteq$ $\left|v_{2}\right| \doteq \frac{1}{2} c\left(E x\left(u_{1},\|v\|\right)+E x\left(u_{2},\|v\|\right)\right)$. There is a sequence $t, t \subseteq v_{1}$ equivalent to $u_{1}$. After deleting all $a$-occurrencess, $a \in S(t)$ from $v_{2}$ we get a $\left\|u_{2}\right\|$-regular subsequence of $v_{2}$ long enough to contain inevitably $u_{2}$. Thus $u_{1} u_{2} \prec v$ which proves the claim.

For a given reducible nonchain sequence $u$ we decompose $u=u_{1} u_{2} \ldots u_{m}$ where $u_{i}$ are irreducible and $S\left(u_{i}\right)$ are mutually disjoint. The previous claim yields $E x(u, n)=$ $O\left(E x\left(u_{1}, n\right)+E x\left(u_{2}, n\right)+\cdots+E x\left(u_{m}, n\right)\right)$. Any term of this sum is a big function and $E x\left(u_{i}, n\right)=O(E x(u, n))(3$. of Fact 2.1). Hence the lemma follows.

Now we are able to continue in the proof. Let $u, v, t$ be as above, $v$ is a nonchain and $w$ is a $\|t\|=\|u\|+\|v\|$ - regular sequence not containing $t$. Let $f:\{1,2, \ldots\} \rightarrow$ $\{1,2, \ldots\}$ be a nondecreasing big function that will be specifized later. We take the leftmost occurrence in $w$ and add occurrence after occurrence maintaining in every step the condition $|s| \leq f(|| s||)$ for currently constructed contiguous subsequence $s$ of $w$. In case we get equality we finish $s$ and start a new $s$ by the next occurrence. On the end we get the decomposition

$$
w=w_{1} w_{2} \ldots w_{h} w_{0},\left|w_{i}\right|=f\left(\| w_{i}||\right), i>0,\left|w_{0}\right| \leq f\left(\left\|w_{0}\right\|\right) .
$$

We need $f(n)$ be sufficiently large but not too much, we need namely:

$(* *) \quad f(n)>\frac{1}{\|v\|}(\|u\|+\|v\|)(E x(v, n)+\|u\|)$ for any $n \geq 1$.

$(* * *) f(n)=O(E x(v, n))$ for $n \geq 1$.

Thus the choice $f(n)=d E x(v, n)$ for a large constant $d$ clearly meets all conditions $(*),\left(^{* *}\right)$ and $(* * *)$. Obviously $f(n)$ is nondecreasing and big because $E x(v, n)$ is. For any $i=1,2, \ldots, h$ let $w_{i}^{*}, w_{i}^{*} \subseteq w_{i}$ be such that $\left\|w_{i}^{*}\right\|=\left|w_{i}^{*}\right|=\left\|w_{i}\right\|$ (we take for any $x \in S\left(w_{i}\right)$ exactly one $x$-occurrences). Thus $\left|w_{i}^{*}\right| \geq 2\|u\|-1$ according to $\left(^{*}\right)$. 
Then we choose $w_{i}^{* *}, w_{i}^{* *} \subseteq w_{i}^{*}(i>0)$ such that $\left|w_{i}^{* *}\right| \geq\left|w_{i}^{*}\right|-(|| u||-1)>\frac{1}{2}\left|w_{i}^{*}\right|$ and that the two sequences

$$
w_{o d d}=w_{1}^{* *} w_{3}^{* *} \ldots w_{h-1}^{* *}, w_{e v e n}=w_{2}^{* *} w_{4}^{* *} \ldots w_{h}^{* *}
$$

(suppose for simplicity $h$ is even) are $\|u\|$-regular. This is achieved by deleting at most $\|u\|-1$ occurrences from $w_{i}^{*}$ which equal to one of the last $\|u\|-1$ occurrencess of $w_{i-2}^{*}$. It suffices to show $w_{\text {odd }} \nsucc u, w_{\text {even }} \nsucc u$. Then conclude:

$$
\begin{gathered}
|w|=\sum_{i=1}^{h}\left|w_{i}\right|+\left|w_{0}\right| \leq \sum_{i=1}^{h} f\left(\left|w_{i}^{*}\right|\right)+f\left(\| w_{0}||\right) \leq \sum_{i=1}^{h} f\left(2\left|w_{i}^{* *}\right|\right)+f\left(\| w_{0}||\right)= \\
=\sum_{i \text { odd }}^{h} f\left(2\left|w_{i}^{* *}\right|\right)+\sum_{i \text { even }}^{h} f\left(2\left|w_{i}^{* *}\right|\right)+f\left(\| w_{0}||\right) \leq
\end{gathered}
$$

(bigness of $f$ )

$$
\leq c f\left(2\left|w_{\text {odd }}\right|\right)+c f\left(2\left|w_{\text {even }}\right|\right)+f(\|w\|) \leq 2 c f(2 E x(u,\|w\|))+f(\|w\|)=
$$

$(\operatorname{property}(* * *))$

$$
=2 c d E x(v, 2 E x(u,\|w\|))+d E x(v,\|w\|)=O(E x(v, 2 E x(u,\|w\|))) .
$$

Suppose now on the contrary that, say, $w_{\text {odd }}$ contains $u$ (the sequence $w_{\text {even }}$ is treated similarily). Thus $u^{*}=u_{1}^{*}\left(a^{*}\right)^{2} u_{2}^{*} \subseteq w_{\text {odd }}$ is equivalent to $u$. The two $a^{*}$-occurrencess must lie in two distinct segments $w_{2 i+1}^{* *}, w_{2 j+1}^{* *}, i<j$ for there is no repetition in any $w_{i}^{* *}$ and hence $u_{1}^{*} a^{*} w_{2 j} a^{*} u_{2}^{*} \subseteq w$. Now we proceed as in the proof of the claim above, we delete all $x$-occurrencess, $x \in S\left(u^{*}\right)$, from $w_{2 j}$ and obtain a $\|v\|$-regular subsequence $w_{2 j}^{\prime}, w_{2 j}^{\prime} \subseteq w_{2 j}$. Clearly

$$
\left|w_{2 j}^{\prime}\right| \geq \frac{\|v\|}{\|u\|+\|v\|}\left|w_{2 j}\right|-\|u\|
$$

which follows from the fact that the deletion of all occurrencess of a symbol from a $k$-regular sequence $s$ yields a $k-1$-regular subsequence $s^{\prime}$ of $s$ of the length at least $\frac{k-1}{k}(|s|-1)$. But then, according to $(* *)$,

$$
\begin{gathered}
\left|w_{2 j}^{\prime}\right|>\frac{\|v\|}{\|u\|+\|v\|} \cdot \frac{\|u\|+\|v\|}{\|v\|}\left(\operatorname{Ex}\left(v,\left\|w_{2 j}\right\|\right)+\|u\|\right)-\|u\|= \\
=E x\left(v,\left\|w_{2 j}\right\|\right) \geq \operatorname{Ex}\left(v,\left\|w_{2 j}^{\prime}\right\|\right)
\end{gathered}
$$

and $v \prec w_{2 j}^{\prime}$. Consequently $t=u_{1} a v a u_{2} \prec w$ which is a contradiction. 


\section{Proof of Theorem B}

This theorem is in some sense stronger than Theorem A because in Theorem B the sequence $v=b^{2 i}$ is split into two parts which are inserted in two places of $u$, in the middle and on the end. In theorem A we put simply the whole $v$ in the middle of $u$. Therefore, one can expect a more complicated proof. We start with three preliminary lemmas.

Lemma 5.1 Let $l \geq 2$ be an integer and let $\varepsilon, 0<\varepsilon<1$ be a constant. Suppose $v$ is a $\left\lceil\frac{l}{\varepsilon}\right\rceil$-regular sequence. Then any $v_{1}, v_{1} \subseteq v,\left|v_{1}\right| \geq \varepsilon|v|$ contains a subsequence $v_{2}, v_{2} \subseteq v_{1}$ such that

1. $v_{2}$ is l-regular

2. $\left|v_{2}\right| \geq\left(\frac{\varepsilon}{l}\right)^{2}|v|$

Proof: Let $v$ and $v_{1}$ be as described. We split $v=w_{1} w_{2} \ldots w_{h} w$ where $\left|w_{i}\right|=$ $\left\lceil\frac{l}{\varepsilon}\right\rceil, i=1,2, \ldots h,|w| \leq\left\lceil\frac{l}{\varepsilon}\right\rceil$. Let

$$
A=\left\{i|| v_{1} \cap w_{i} \mid \leq l-1\right\}, a=|A|, B=\left\{i|| v_{1} \cap w_{i} \mid \geq l\right\}, b=|B| .
$$

Clearly

$$
a+b=h=\left\lfloor\frac{|v|}{\left\lceil\frac{l}{\varepsilon}\right\rceil}\right\rfloor \leq \frac{\varepsilon}{l}|v| .
$$

Further $a(l-1)+b\left\lceil\frac{l}{\varepsilon}\right\rceil \geq\left|v_{1}\right| \geq \varepsilon|v|$. Hence $\varepsilon|v| \leq\left(\frac{\varepsilon}{l}|v|-b\right)(l-1)+b\left\lceil\frac{l}{\varepsilon}\right\rceil$ and

$$
b \geq \frac{\frac{\varepsilon}{l}|v|}{\left\lceil\frac{l}{\varepsilon}\right\rceil-l+1} \geq\left(\frac{\varepsilon}{l}\right)^{2}|v| .
$$

By appropriately taking one occurrences from any $v_{1} \cap w_{i}, i \in B$ we create an $l$ regular subsequence $v_{2}, v_{2} \subseteq v_{1},\left|v_{2}\right|=b$.

Suppose $u=a_{1} a_{2} \ldots a_{m}$ is a sequence. An interval $I=\left\langle a_{j}, a_{j+k}\right\rangle$ in $u$ is any contiguous subsequence $a_{j} a_{j+1} \ldots a_{j+k}, k \geq 1$ of length at least 2 . In the case $a_{j}$ and $a_{j+k}$ are both $a$-occurrences we call $I$ an $a$-interval. An ordered sequence $(u,<)$ is a sequence enriched by a linear order $(S(u),<)$.

Let $(u,<), u=a_{1} a_{2} \ldots a_{m}$ be an ordered sequence, $a_{i}$ be an $a$-occurrences in $u$ $\left(a \in S(u)\right.$ ) and $c, d \geq 1$ be two integers. We say that $a_{i}$ is $(c, d)$-covered (in $u$ ) if there is an interval $I$ in $u$ such that

1. $a_{i} \in I$

2. there are at most $c$ a-occurrencess in $I$

3. there are $2 d$ occurrencess of $d$ (not necessarily distinct) symbols $x_{j} \in S(u), j=$ $1 \ldots d, x_{j}<a$ in $I$, each of these symbols occurs at least twice in $I$. 
Lemma 5.2 For any 2-regular ordered sequence $(u,<)$ and any integer $r \geq 2$ either $|u| \leq 720 r\|u\|$ or there are at least $\frac{1}{10}|u|$ occurrencess in $u$ which are $(8 r, r-1)$ covered.

Proof: We can suppose that $S(u)=\{1,2, \ldots, n\}$ and that $\mathbf{i}$ coincides with the standard order of integers. We will define by induction sets $U_{0}, U_{1}, \ldots, U_{n}$ of disjoint intervals in $u$. For any $j=1,2, \ldots, n$ the set $U_{j}$ will contain some $k$-intervals, $k=1,2, \ldots, j$. First put $U_{0}=\emptyset$. Fix $j$ and suppose that the set $U_{j-1}$ have been defined. We split all $j$-occurrencess in $u$ in $m 8 r$-tuples $T_{1}, T_{2}, \ldots T_{m}$ and a residual tuple $T$ of the size at most $8 r-1$ so that $T_{1}$ consists of the $8 r$ leftmost $j$-occurrencess, $T_{2}$ consists of the next $8 r j$-occurrencess etc. Define

$$
\mathcal{S}_{j}=\left\{i \mid \text { at least one } j \text {-occurrences of } T_{i} \text { is not }(8 r, r-1) \text {-covered }\right\} \text {. }
$$

The elements of $T_{i}, i \in \mathcal{S}_{j}$ group in $4 r$ pairs $\left(x, x^{\prime}\right)$ of consecutive elements generating $j$-intervals $\left\langle x, x^{\prime}\right\rangle$. The set $U_{j}$ consists of all those intervals $\left\langle x, x^{\prime}\right\rangle$ and of all members of $U_{j-1}$ not intersecting them. Now, crucially, $4 r$ intervals $\left\langle x, x^{\prime}\right\rangle$ corresponding to one $T_{i}, i \in \mathcal{S}_{j}$ intersect all together at most $(r-2)+2=r$ intervals of $U_{j-1}$. This holds by the definition of $\mathcal{S}_{j}$. Thus

$$
\left|U_{j}\right| \geq 4 r\left|\mathcal{S}_{j}\right|+\left|U_{j-1}\right|-r\left|\mathcal{S}_{j}\right|=3 r\left|\mathcal{S}_{j}\right|+\left|U_{j-1}\right|
$$

and

$$
\left|U_{n}\right| \geq 3 r \sum_{j=1}^{n}\left|\mathcal{S}_{j}\right|
$$

Since $u$ is 2-regular and $U_{n}$ consists of disjoint $k$-intervals,

$$
|u| \geq 3\left|U_{n}\right| \geq 9 r \sum_{j=1}^{n}\left|\mathcal{S}_{j}\right|
$$

The number of occurrencess in $u$ which are $(8 r, r-1)$-covered is therefore at least ( suppose $|u| \geq 720 \mathrm{rn}$ )

$$
|u|-\sum_{j=1}^{n} 8 r\left|\mathcal{S}_{j}\right|-(8 r-1) n \geq|u|-\frac{8 r}{9 r}|u|-\frac{1}{90}|u|=\frac{1}{10}|u| .
$$

In the remaining lemma we force the symbols $x_{j}$ to be distinct. Suppose $(u,<)$, $u=a_{1} a_{2} \ldots a_{m}$ is an ordered sequence, $v \subseteq u$ is a subsequence, $y \in v$ is an $a$ occurrences $(a \in S(u))$ and $c, d \geq 1$ are two integers. We say that $y$ is strongly $(v, c, d)$-covered if there is an interval $I$ in $u$ such that

1. $y \in I$ 
2. there are at most $c$ a-occurrencess in $I \cap v$

3. there are $2 d$ occurrencess of $d$ distinct symbols $x_{j} \in S(u), j=1 \ldots d, x_{j}<a$ in $I$, any of these symbols occurs at least twice in $I$.

Lemma 5.3 Suppose two integers $k \geq 2, d \geq 1$ are given. Then there exist two integers $l \geq 2, c \geq 1$ and two positive constants $\varepsilon, \Delta>0$ such that for any l-regular ordered sequence $(u,<)$ one of the following statements holds.

1. $|u| \leq \Delta\|u\|$

2. there is a k-regular subsequence $v, v \subseteq u,|v| \geq \varepsilon|u|$ such that any occurrence in $v$ is strongly $(v, c, d)$-covered.

Proof: We proceed by induction on $d$. To prove the statement for $d=1$ we take a $10 k$-regular ordered sequence $(u,<)$. Then, according to the previous lemma $(r=2)$, either $|u| \leq 1440|| u \|$ or there is a subsequence $v \subseteq u,|v| \geq \frac{1}{10}|u|$ whose each element is in $u(16,1)$-covered. We apply Lemma 5.1 and choose a $k$-regular subsequence $v^{\prime} \subseteq v,\left|v^{\prime}\right| \geq\left(\frac{1}{10 k}\right)^{2}|u|$. Any element of this sequence is still $(16,1)$-covered. We see that for $k \geq 2, d=1$ we can put $c=16, l=10 k, \Delta=1440$ and $\varepsilon=\left(\frac{1}{10 k}\right)^{2}$.

Suppose the statement has been proved for $d>1$ and any $k \geq 2$. Our task is to derive the existence of the numbers $c(k, d+1), l(k, d+1), \Delta(k, d+1)$ and $\varepsilon(k, d+1)$ corresponding to a given $d+1, k$. We show that it is possible to take

$$
l(k, d+1)=l(10 k, d), c(k, d+1)=8 r, \varepsilon(k, d+1)=\left(\frac{1}{10 k}\right)^{2} \varepsilon(10 k, d)
$$

and

$$
\Delta(k, d+1)=\max \left(\Delta(10 k, d), \frac{720 r}{\varepsilon(10 k, d)}\right)
$$

where

$$
r=d . c(10 k, d)+2 .
$$

Suppose $(u,<)$ is an ordered $l(k, d+1)$-regular sequence. Then, according to the induction hypothesis, either $|u| \leq \Delta(10 k, d)\|u\|$ or there is a $10 k$-regular subsequence $v_{1}, v_{1} \subseteq u,\left|v_{1}\right| \geq \varepsilon(10 k, d)|u|$ whose each element is strongly $\left(v_{1}, c(10 k, d), d\right)$ covered. Suppose the latter possibility holds.

Now, according to Lemma 5.2, either $\left|v_{1}\right| \leq 720 r\left\|v_{1}\right\|$ or there is a subsequence $v_{2}, v_{2} \subseteq v_{1},\left|v_{2}\right| \geq \frac{1}{10}\left|v_{1}\right|$ any element of which is $(8 r, r-1)$-covered in $v_{1}$. Suppose the latter possibility holds.

Finally, we choose according to Lemma 5.1 a $k$-regular subsequence $v_{3}, v_{3} \subseteq v_{2}$ where $\left|v_{3}\right| \geq\left(\frac{1}{10 k}\right)^{2}\left|v_{1}\right|$. We prove that any element of $v_{3}$ is strongly $\left(v_{3}, c(k, d+1), d+1\right)$ covered. Let $x \in v_{3}$ be an $a$-occurrences. According to the choice of $v_{2}$ there is an interval $I$ in $v_{1}$ that $(8 r, r-1)$-covers $x$. This implies that there are $r-1$ symbols $a_{j} \in S(u), j=1,2, \ldots r-1, a_{j}<a$ any of which occurs twice in $I$ and that there are at most $8 r$ a-occurrences in $I$. 
We define $I^{u}$ as the interval in $u$ spanned by $I$ and show that $I^{u}$ strongly $\left(v_{3}, c(k, d+\right.$ $1), d+1$ )-covers $x$. Property 1 . is obvious, property 2 . requires that $I^{u} \cap v_{3}$ contains at most $c(k, d+1)$ a-occurrences which is true already for $I=I^{u} \cap v_{1}$. It remains to show that 3 . holds.

In case at least $d+1$ symbols $a_{j}$ are distinct we are finished. Otherwise (see the definition of $r$ ) some symbol, say $a_{1}$, has at least $2 c(10 k, d)+1$ occurrencess in $I^{u}$. Denote the subsequence consisting of these occurrencess as $p$. Now we make use of the choice of $v_{1}$ from $u$. We denote by $J$ the interval in $u$ which strongly $\left(v_{1}, c(10 k, d), d\right)$-covers the middle element of $p$. Clearly $J \subseteq I^{u}$. We see that there are again $d+1$ distinct symbols satisfying 3 . for $I^{u}$. Namely $a_{1}$ and those $d$ symbols less then $a_{1}$ any of which occurs twice in $J$. In the former possibilitties it is easy to check that $|u| \leq \Delta(k, d+1)$.

Now we are able to prove Theorem B. Suppose $u=u_{1} a^{2} u_{2} a$ is a sequence, $b \notin S(u)$ is a new symbol and $i \geq 1$ is an integer. Our task is to prove $E x\left(u_{1} a b^{i} a u_{2} a b^{i}, n\right)=$ $O(E x(u, n))$. The lower bound $E x\left(u_{1} a b^{i} a u_{2} a b^{i}, n\right)=\Omega(E x(u, n))$ follows from $u \prec$ $u_{1} a b^{i} a u_{2} a b^{i}$. By Fact 2.2 it suffices to prove $E x(t, n)=O(E x(u, n))$ where $t=$ $u_{1} a b^{2} a u_{2} a b$. Put $k=d=\|u\|$ in the previous lemma and let $c, l, \varepsilon$ and $\Delta$ be the corresponding constants guaranteed by this lemma.

Suppose the sequence $w$ is $l$-regular and does not contain $t$. We define the linear order $(S(w),<)$ by $a<b$ iff the rightmost $a$-occurrences lies to the right of the rightmost $b$-occurrences. In the first case of the previous lemma $|w| \leq \Delta\|w\|$. Otherwise there is a ||$u \|$-regular subsequence $v, v \subseteq w,|v| \geq \varepsilon|w|$ whose each element is strongly $(v, c,\|u\|)$-covered. We show that $v$ does not contain the sequence $u^{\prime}=u_{1} a^{2 c+1} u_{2} a$. Suppose on the contrary that $s=u_{1}^{*}\left(a^{*}\right)^{2 c+1} u_{2}^{*} a^{*}, s \subseteq v$ is equivalent to $u^{\prime}$. We denote by $p$ the subsequence $\left(a^{*}\right)^{2 c+1}$ of $s$. The middle $a^{*}$-occurrences in the subsequence $p$ must be strongly $(v, c,\|u\|)$-covered by an interval $I$ in $w$. Let $J$ be interval in $w$ spanned by the first and by the last $a^{*}$-occurrences in $p$. Clearly $I \subseteq J$. There are $\|u\|$ distinct symbols $x_{i}, x_{i}<a^{*}, i=1,2, \ldots\|u\|$ and each of them occurs at least twice in $I$. By the definition of $;$ each of these symbols occurs to the right of $s$. At least one of them is not an element of $S(s)$ and we get $t \prec v$ which is a contradiction. Thus $u^{\prime} \nprec v$ and $|v| \leq E x\left(u^{\prime},\|v\|\right) \leq g E x(u,\|v\|)$ for some constant $g$ according to Fact 2.2. Hence

$$
|w| \leq \frac{1}{\varepsilon}|v| \leq \frac{g}{\varepsilon} \operatorname{Ex}(u,\|v\|) \leq \frac{g}{\varepsilon} E x(u,\|w\|) .
$$

Together

$$
|w| \leq \max \left\{\Delta\|w\|, \frac{g}{\varepsilon} \operatorname{Ex}(u,\|w\|)\right\}=O(E x(u,\|w\|)) .
$$

We have proved that

$$
E x(t, n, l)=O(E x(u, n)) .
$$

Finally, according to 2 . of Fact 2.1, the estimate

$$
E x(t, n)=E x(t, n,\|t\|)=O(E x(t, n, l))=O(E x(u, n))
$$


holds. The Theorem is proved.

\section{Acknowledgement}

The authors are grateful to Jaroslav Nešetřil and to Jiří Matoušek for their fruitful comments.

(This paper is to appear in Combinatorica.)

\section{References}

[1] R. Adamec, M. Klazar and P. Valtr, Generalized Davenport-Schinzel sequences with linear upper bound, . Discrete Math. (108), 1992219-229

[2] P. K. Agarwal, Intersection and decomposition algorithms for planar arrangements, Cambridge University Press 1991.

[3] P. K. Agarwal, M. Sharir and P. Shor, Sharp upper and lower bounds on the length of general Davenport-Schinzel sequences, J. Comb. Theory A 52 (1989), $228-274$.

[4] H. Davenport and A. Schinzel, A combinatorial problem connected with differential equations, Amer. J. Math. 87 (1965), 684-689.

[5] H. Davenport, A combinatorial problem connected with differential equations II, Acta Arith. 17 (1971), 363-372.

[6] Z. Füredi and P. Hajnal, Davenport-Schinzel theory of matrices, Discrete Math. 103 (1992), 233-251.

[7] S. Hart and M. Sharir, Nonlinearity of Davenport-Schinzel sequences and of generalized path compression schemes, Combinatorica 6 (1986), 151-177.

[8] M. Klazar, A general upper bound in Extremal theory of sequences, Comment. Math. Un. Carolinae 33 (1992), 737-746.

[9] M. Klazar, A linear upper bound in Extremal theory of sequences (to appear in J. of Comb. Th. A).

[10] M. Klazar, Two results on a partial ordering of finite sequences (to appear in Comment. Math. Un. Carolinae).

[11] P. Komjath, A simplified construction of nonlinear Davenport-Schinzel sequences, J. Comb. Th. A 49 (1988), 262-267.

[12] M. Sharir, Almost linear upper bounds on the length of general DavenportSchinzel sequences, Combinatorica 7 (1987), 131-143. 
[13] M. Sharir and P. K. Agarwal, Davenport-Schinzel sequences and their geometric applications, manuscript 1993.

[14] E. SzmerÉDI, On a problem by Davenport and Schinzel, Acta Arith. 25 (1974), 213-224.

[15] A. Wiernik and M. Sharir, Planar realization of nonlinear Davenport-Schinzel sequences by segments, Discrete Comput. Geometry 3 (1988), 15-47 